\title{
Online Microscope Simulators for Training and Outreach
}

\author{
Caroline Fuery ${ }^{1}$, Jenny Whiting ${ }^{1}$, Bronwen Cribb $^{2}$, John Williams ${ }^{3}$ and Julie Cairney ${ }^{1,4}$
}

${ }^{1 .}$ Australian Microscopy and Microanalysis Research Facility, Madsen Building F09, The University of Sydney, NSW, 2006, Australia.

2. Centre for Microscopy and Microanalysis, The University of Queensland, Brisbane, QLD, 4072, Australia

3. FEI, 5350 NE Dawson Creek Drive Hillsboro, Oregon 97124 USA.

4. Aerospace, Mechanical and Mechatronic Engineering, The University of Sydney, Sydney, NSW, 2006, Australia.

The Australian Microscopy and Microanalysis Research Facility (AMMRF) has developed a suite of online learning tools, involving sophisticated microscope simulators in modules suited to training of microscopy users and STEM outreach to school students.

The AMMRF a national grid of equipment, instrumentation and expertise in microscopy and microanalysis. It is a collaborative distributed network of core facilities that provides nanostructural characterisation capability and services to more than 3,000 researchers annually. Once a researcher has identified the centre they need to work with, they are supported through a common research experience. This comprises stages to define and formulate the scientific question, identify the most appropriate techniques, register the project and meet to discuss the techniques, plan training and arrange access to instrumentation. Users then go on to data acquisition and analysis, data management and ultimately an outcome such as a research publication, grant application or invention registration or patent. With more than 3,000 users annually, training can take up a significant portion of an instrument's bookable time, making it unavailable for experienced researchers. There was therefore a strong need to efficiently train users while minimising time taken up with on-instrument training. Online training tools that incorporate instrument simulators were developed in response to this need. They have proven highly beneficial in reducing on-instrument training time while turning out more competent and knowledgeable users. The free, online site, MyScope ${ }^{\mathrm{Tm}}$, covers six microscopy and microanalytical techniques and has reduced the on-instrument training time by around $25 \%$ [1].

The modules provide a novel advancement in online training with an ecosystem composed of a supported learning environment for flexible learning; a tailoring capability for academics and trainers; self guided tutorials with videos, animations and glossary to prepare students with knowledge and specialist language; instrument simulators where they can practice use of instrumentation; and online competency testing to demonstrate readiness for hands-on experience [1]. The instrument simulators were specifically designed to provide a realistic interface that users can master at their own pace before presenting for one-on-one training. This builds confidence and competency.

MyScope $^{\mathrm{TM}}$ is supported by TechFi ${ }^{\mathrm{Tm}}$, a web application that enables prospective facility users to identify the most suitable techniques for their research, based on a researcher-centric approach and terminology. It offers one area for biological scientists and another for researchers in the physical sciences, which allow them to identify techniques based on research dimensions in corresponding fields. Locations and contact details to each of the AMMRF facilities invite users to get started immediately.

As well as training AMMRF microscope users, MyScope ${ }^{\mathrm{TM}}$ has been taken up by other core facilities and 
educators around the world. Well over 100,000 users per year access the site, clocking up nearly 1 million page views.

The success of MyScope ${ }^{\mathrm{Tm}}$ led to a partnership between the AMMRF and FEI Company. This collaboration aimed to support FEI's mission to provide an online STEM engagement resource for school-aged children. MyScope ${ }^{\mathrm{TM}}$ Outreach was the result, based on the scanning electron microscopy (SEM) module in MyScope ${ }^{\mathrm{Tm}}$. This popular site encourages discovery-based learning through guided exploration on the SEM simulator and is an ideal tool for use at home, in the classroom and in outreach venues such as museums. The simulator has many of the same features as the original MyScope ${ }^{\mathrm{TM}} \mathrm{SEM}$ simulator and the users can learn about both the technicalities of operating an SEM and make discoveries by exploring a range of 68 authentic themed samples with the simulator. It also has background information on basic microscopy, how an SEM works and a variety of supporting activities in the areas of biology, chemistry, physics, mathematics and communications.

MyScope $^{\mathrm{TM}}$ Outreach has many additional advantages for educators teaching microscopy. Many schools do not have access to even a basic light microscope and for those that do it can be hard to provide a consistent learning experience. The high quality electron microscope images in MyScope ${ }^{\mathrm{TM}}$ Outreach make discovery exciting and enable visualisation of the micro- and nanoscale world, where much of modern science takes place. It is also an excellent learning activity for remote schools with little access to excursions and other external resources.

Observations of children leave us in no doubt of how engaged they are by use of the simulator [2]. It generates a sense of awe and wonder in the learner as they make discoveries. The complexity of the controls also generates a sense of achievement in knowing how to use the microscope and provides an experience that parallels the fun of playing a video game. It is also highly valued by teachers [2].

Through our online learning tools MyScope ${ }^{\mathrm{Tm}}$ and MyScope ${ }^{\mathrm{Tm}}$ Outreach, instrument simulators are playing a valuable role in education and training for both professional researchers, school students and the public generally.

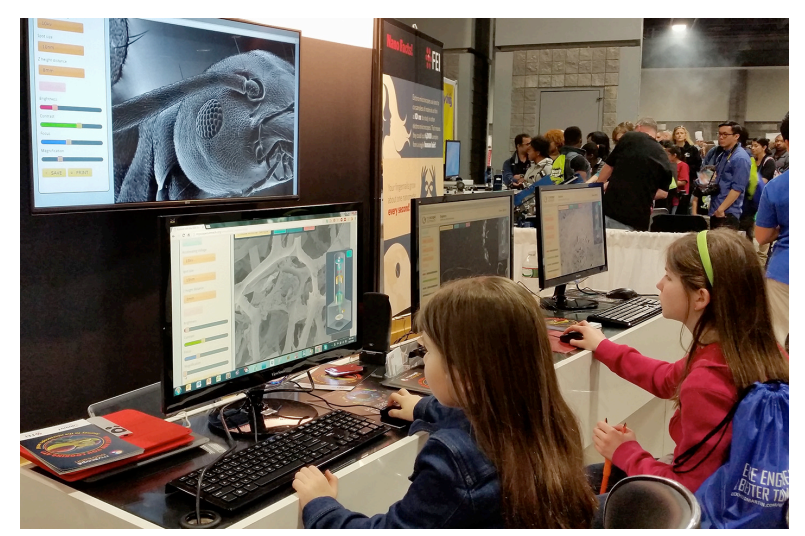

Children using MyScope Outreach at a science festival

References:

[1] B Cribb et al., Online Education and Training for Microscopy and Microanalysis: MyScope ${ }^{\mathbf{T M}}$, Microscopy Today, Volume 24 (4), July 2016, pp 44 - 49

[2] AMMRF Profile 2016, p17. Available at: ammrf.org.au. 